\title{
FREDI: Robust RSS-based Ranging with Multipath Effect and Radio Interference
}

\author{
Yu Zhao ${ }^{\ddagger}$, Yunhuai Liu ${ }^{\ddagger}$, Tian $\mathrm{He}^{*}$, Athanasios V. Vasilakos ${ }^{\dagger}$, and Chuanping $\mathrm{Hu}^{\ddagger}$ \\ $\ddagger$ The Third Research Institute of Ministry of Public Security, P.R.China \\ *University of Minnesota, U.S. \\ ${ }^{\dagger}$ Kuwait University, Kuwait \\ Email:\{yu.zhao,yunhuai,cphu\}@trimps.ac.cn, tianhe@cs.umn.edu, vasilako@ath.forthnet.gr
}

\begin{abstract}
Radio Signal Strength (RSS) based ranging is attractive by the low cost and easy deployment. In real environments, its accuracy is severely affected by the multipath effect and the external radio interference. The well-known fingerprint approaches can deal with the issues but introduce too much overhead in dynamic environments. In this paper, we attempt to address the issue along a completely different direction. We propose a new ranging framework called Fredi that exploits the frequency diversity to overcome the multi-path effect solely based on RSS measurements. We design a Discrete Fourier Transformation based algorithm and prove that it has the optimal solution under ideal cases. We further revise the algorithm to be robust to the measurement noises in practice. We implement Fredi on top of the USRP-2 platform and conduct extensive real environments in indoor environments. Experimental results show the superiority performance compared with the traditional methods.
\end{abstract}

\section{INTRODUCTION}

Ranging is crucial for precise localizations. With an accurate ranging technology, localizations can be easily obtained by trilateration [11]. Among the various ranging techniques, Radio Signal Strength (RSS) based approaches are quite attractive because of the low cost and easy deployment. Nodes simply measure the pairwise link RSS and then compute the corresponding distance between the sender and receiver.

In an ideal open-space environment, there is a unique monotonic function between RSS and the physical distance which can help to accomplish the computation. In real environments, however, the RSS is severely affected by various physical phenomenon such as the multipath effects and external interference, resulting complex functions of many free parameters. As RSS measurements are the only input during the ranging, accurate RSS-based ranging is a very challenging task.

In recent years, frequency diversity has been widely studied for wireless communications (e.g., FARA [7]). By frequency diversity, a given pair of nodes may have very different RSS values at different frequencies, which is mainly due to the multipath effect. When there are different paths, waves of different paths will combine constructively or destructively depending on their phases. As the phase is a function of the frequency, the combination of waves will be different at different frequencies, resulting in changed RSS values. By carefully analyzing such RSS differences, we can obtain the phase information, and accordingly compute the distance. For ease of presentation, we name the general framework as Fredi.
When analyzing the RSS values, we find that the RSS value model function has a very special structure of trigonometric components (e.g., sine and cosine functions). For such a structure, Discrete Fourier Transformation (DFT) is a powerful tool which is originally used for signal processing. Based on DFT, we design algorithms and prove that in the ideal case, the solutions are accurate. We further find that in real environments these algorithms do not perform well because of the measurement noises. We also design a revised DFT-based algorithm which provides robustness to some measurement input noises.

Compared with the traditional RSS-based ranging techniques, Fredi mainly poses following advantages. It does not require any special hardware and no physical layer information is needed. The only input is the RSS, and the only requirement is the fast frequency hopping. When ranging, only the transmitter and receiver are involved and no other cooperation is needed. Fredi also is highly dynamic adaptive and error robustness. During our experiments people can freely move around.

The main contributions of this paper are as follows:

First, to the best of our knowledge, we are the first to address the multi-path effect, measurement errors in RSSbased ranging solely based on RSS measurements.

Second, we design a novel DFT-based algorithm and prove that the algorithm can produce exact solutions in the ideal case. We further design a robust DFT-based algorithm to tolerate some measurement noises.

Third, we implement the proposed algorithms on top of the USRP2 platform and conduct comprehensive indoor experiments.

The rest of the paper will be organized as follows. In Sec. 2, we start from the background of the Fredi ranging including the motivation and the Fredi's framework. In Sec. 3, we show how to solve the problem by Fredi algorithm. We give analysis on Fredi and conduct performance evaluation in Sec. 4. In the last section, we draw conclusions.

\section{BACKGROUND}

In this section, we give some background information including the insight of the frequency diversity for RSS-based ranging and Fredi general framework. 


\section{A. Motivation}

Fredi is in-sighted by FARA [7], a rate adaption algorithm that exploits frequency diversity to improve wireless communication efficiency. Because of the frequency diversity, a pair of nodes may have very different RSS values at nearby frequencies. Frequency diversity is mainly due to the multipath effect in which the radio waves will traverse through different physical paths and combine at the receiver. Such RSS difference carries the path information, which can be explored to figure out all the dynamic information.

Suppose there are two radio paths $a$ and $b$. Waves are featured by two variables, the amplitude and the phase. At each frequency, because of the different path lengths, there will be a phase difference $\frac{d_{a}-d_{b}}{\lambda}$ between waves of different paths. And at different frequencies, this phase difference will also be different, i.e., $\frac{d_{a}-d_{b}}{\lambda_{1}} \neq \frac{d_{a}-d_{b}}{\lambda_{2}}$, resulting in changed RSS values. By exploring such different $R S S$, we can build equations to figure the path length $d_{a}$ out.

In the next subsection, we give a general framework of Fredi to exploit the frequency diversity.

\section{B. Fredi general framework}

Compared with the standard RSS-based ranging, Fredi simply adds one more operation. After each RSS measurement, the transmitter and receiver should switch to another frequency and collect the new RSS value. As long as all supported frequencies are measured, we can make the computation. Supposing there are $N$ different frequencies, we thus can have up to $N$ RSS measurements, denoted as $\hat{s}_{n}, n \leq N$. Our goal is to figure out all the dynamic factors that fit these measurements, i.e.,

$$
\begin{aligned}
& \text { Input : } \lambda_{n}, \hat{s}_{n}, n \leq N \\
& \text { Output : } d_{1}
\end{aligned}
$$

where $d_{1}$ is the physical distance between the transmitter and receiver. Mathematically this is a curvature fitting problem that belongs to non-linear optimization category. We however find that such problem has no standard methods and needs a customized solution.

In the next we will present our DFT-based solution. To ease the presentation, we will start from analyzing the simplest 1path scenario, i.e., there is only one path between nodes, and gradually approach the most general case, i.e., there are more number of paths, and measurements contain errors.

\section{IDEAL SCENARIOS}

In this section, we present the DFT-based algorithm to solve the problem. We start from the simplest one-path scenario, and extend to the two-path, three-path, and finally to the general M-path scenario. In this section, we assume the number of paths $M$ is known and there is no measurement error.

\section{A. One-path Scenario}

In the simplest one-path scenario, the solution is quite straightforward. Radio wave propagations follow the physical laws which are the foundation of wireless communications.
During the propagations, radio wave energy field will fade out. Consider a simple sine radio wave. When the waves directly propagate to the receiver, a Line-of-sight (LoS) path is established. The LoS path propagations can be modeled by the Friss open space model where the energy field strength is

$$
E_{L o S}(t)=\frac{\sqrt{G_{t} G_{r} P_{r}}}{\sqrt{4 \pi} d} \sin \left(\frac{2 \pi c}{\lambda} t+2 \pi \frac{d}{\lambda}\right)
$$

Here $G_{t}$ is the gain of the sender, $G_{r}$ is the receiver gain, $P_{r}$ is the transmission power, $\lambda$ is the radio wavelength, $c$ is the light speed, $d$ is the LoS path length and $t$ is the time. According to Equ. 1, under the single path scenario, the RSS at different frequencies $\hat{s}_{n}$ is a constant. Thus, the LoS path length $d_{1}$, also the physical distance between the transmitter and receiver, can be easily obtained by

$$
\frac{G_{t} G_{r} P_{r}}{8 \pi d^{2}}=\hat{s}_{n}
$$

Here $G_{t}, G_{r}, P_{r}$ are hardware-dependent that can be obtained in an offline manner. Since this is a single-run operation, the overhead is acceptable.

\section{B. Two-path Scenario}

In this part, we first introduce the physical laws of multipath effect, and then derive the system model for the two-path scenario and propose our DFT-based solution.

1) Multi-path effect: In practical environments, there can be two or more physical radio propagation paths from the transmitter to the receiver, referred to as multi-path phenomenon. The causes of the multi-path effect are many, such as the atmospheric duct, refraction and reflection. Besides the LoS path, the other physical paths are called Non-Line-of-Sight (NLoS) path.

NLoS paths change in direction of the signal waves at an interface between two different media, resulting in the returned wave to the original medium. In the indoor environments, the reflections typically occur at the surface of the objects, walls, and the ground. With each reflection, partial energy will be the transmittance, partial will be reflected, and the left is absorbed by the medium. An NLoS path will introduce the energy field as

$$
E_{N L o S}(t)=\Gamma \frac{\sqrt{G_{t} G_{r} P_{r}}}{\sqrt{4 \pi} d} \sin \left(\frac{2 \pi c}{\lambda} t+2 \pi \frac{d}{\lambda}\right)
$$

where $\Gamma \in(0,1)$ is a reflection coefficient. Refraction is another source of the NLoS paths. It has a similar effect and thus can also be modeled by Equ. (2). Notice that Equ. (2) can also express LoS path with $\Gamma=1$.

Let $d_{1}$ be the LoS path and $d_{2}$ the NLoS one. When waves arrive at the receiver through the two paths, their signals will be added, and the RSS is the averaged power of the received signal, i.e.,

$$
\begin{aligned}
s(\lambda) & =C \lambda^{2} \sum_{t=1}^{N} \frac{\left(E_{1}(t)+E_{2}(t)\right)^{2}}{N} \\
& =C \lambda^{2}\left(\frac{1}{2 d_{1}^{2}}+\frac{\Gamma_{2}^{2}}{2 d_{2}^{2}}+\frac{\Gamma_{2}}{d_{1} d_{2}} \cos \left(\frac{d_{1}-d_{2}}{\lambda}\right)\right)
\end{aligned}
$$



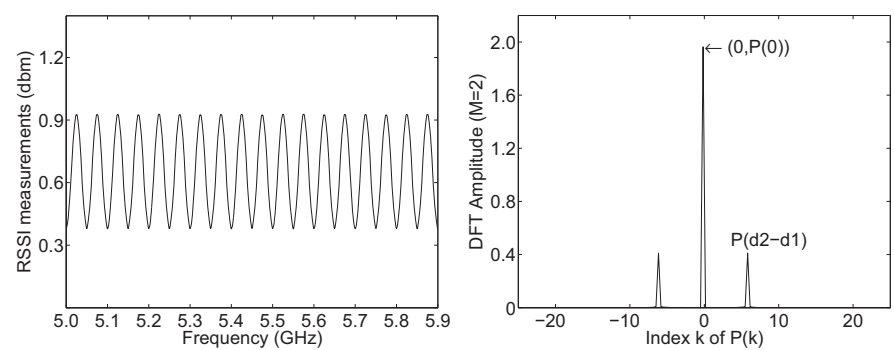

Fig. 1. A two-path scenario with $d_{1}=4, d_{2}=10$, and $\Gamma=0.5$, and its DFT amplitudes; there are two non-negative values, $P(0)$ and $P\left(d_{1}-d_{2}\right)$

where $C=\frac{G_{t} G_{r} P_{r}}{4 \pi}$ is a hardware-dependent constant that can be obtained in an offline manner, $E_{1}$ is the energy field of the LoS path and $E_{2}$ is of the NLoS path. As the convention, $s(\lambda)$ in Equ. (3) is called model function.

Fig. 1 gives an example of Eq. (3). This is a typical trigonometric function that exhibits periodical properties. It has the periodical part $\cos \left(\frac{d_{1}-d_{2}}{\lambda}\right)$ determined by the two path length difference $d_{1}-d_{2}$ and the wave length $\lambda$.

2) DFT-based solution: Fourier analysis is a mathematical tool that helps to find periodic properties of a function. It decomposes the function into its constituent frequencies, and the amplitudes at the frequencies will reflect the periodic strength at the corresponding frequencies. Notice that this "frequency" is not the same frequency of radio waves in wireless communications. Fourier analysis has been widely used in many fields and is particularly useful for functions with trigonometric components like Equ. (3).

Discrete Fourier Transformation (DFT) is a discredited method of Fourier analysis. Given a sequence of $N$ discrete values $\frac{\hat{s}_{n}}{c \cdot \lambda_{n}^{2}}$, DFT transforms them into another sequence of $N$ numbers by the formula:

$$
P(k)=\sum_{n=1}^{N} \frac{\hat{s}_{n}}{C \lambda_{n}^{2}} \cdot e^{-i 2 \pi \frac{k}{N} n}, k=1, \ldots, N
$$

As $P(k)$ is an even function with respect to $k$, in the remainder of this paper we consider only the positive part of $P(k)$, i.e., $k>0$. The $P(0)$ will be explicitly identified besides $P(k)$. We call $k$ the $P(k)$ 's index, and $P(k)$ the DFT amplitude indexed by $\mathrm{k}$.

Applying Equ. (4) on Equ. (3), we have $P(0)=\frac{1}{2 d_{1}^{2}}+$ $\frac{\Gamma_{2}^{2}}{2 d_{2}^{2}}$ and $P\left(d_{1}-d_{2}\right)=\frac{\Gamma_{2}}{d_{1} d_{2}}$. Let $P_{i}$ be the i-th largest DFT amplitude among all $P(k)$, i.e., $P_{1}=\max (P(k))$ and so on, and $\underset{k}{\arg }\left(P_{1}\right)$ be the index of $P_{1}$, i.e., $P\left(\underset{k}{\arg }\left(P_{1}\right)\right)=P_{1}$. In two-path cases, there is only one non-zero $P(k)$, and thus $P\left(d_{1}-d_{2}\right)=P_{1}$. As such, we rewrite the equations as

$$
\left\{\begin{aligned}
\frac{1}{2 d_{1}^{2}}+\frac{\Gamma_{2}^{2}}{2 d_{2}^{2}} & =P(0) \\
\frac{\Gamma_{2}}{d_{1} \cdot d_{2}} & =P_{1} \\
d_{2}-d_{1} & =\underset{k}{\arg \left(P_{1}\right)}
\end{aligned}\right.
$$

Here we have three unknowns $d_{1}, d_{2}, \Gamma_{2}$ and three simple equations. The solution is straightforward and omitted.
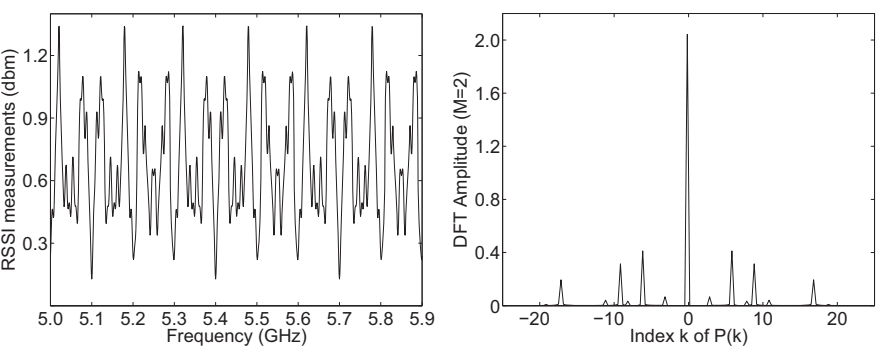

Fig. 2. An M-path case with $d_{1}=4, d_{2}=10, d_{3}=13, d_{4}=21, d_{5}=40$, all $\Gamma=0.5$, and its DFT amplitudes; there are 10 non-negative $P(k)$

\section{Three-path case}

Three-path cases are more complex than the two-path cases. When there are three paths, the model function $s(\lambda)$ is

$$
\begin{aligned}
s(\lambda) & =C \lambda^{2} \sum_{t=1}^{N} \frac{\left(E_{1}(t)+E_{2}(t)+E_{3}(t)\right)^{2}}{N} \\
& =C \lambda^{2}\left(\frac{1}{2 d_{1}^{2}}+\frac{\Gamma_{2}^{2}}{2 d_{2}^{2}}+\frac{\Gamma_{3}^{2}}{2 d_{3}^{2}}+\frac{\Gamma_{2}}{d_{1} d_{2}} \cos \left(\frac{d_{1}-d_{2}}{\lambda}\right)\right. \\
& \left.+\frac{\Gamma_{3}}{d_{1} d_{3}} \cos \left(\frac{d_{1}-d_{3}}{\lambda}\right)+\frac{\Gamma_{2} \Gamma_{3}}{d_{2} d_{3}} \cos \left(\frac{d_{2}-d_{3}}{\lambda}\right)\right)
\end{aligned}
$$

Without lose of generality, assume $\frac{\Gamma_{2}}{d_{2}} \geq \frac{\Gamma_{3}}{d_{3}}$. Applying the DFT method on this model function $s(\lambda)$, we have (indeed, we have more equations but do not need them here)

$$
P_{1}=\frac{\Gamma_{2}}{d_{1} d_{2}}, \quad P_{2}=\frac{\Gamma_{3}}{d_{1} d_{3}}, \quad P_{3}=\frac{\Gamma_{2} \Gamma_{3}}{d_{2} d_{3}}
$$

where $P_{i}$ is the $\mathrm{i}$-th largest $P(k)$. Noticing that

$$
\frac{P_{3}}{P_{1} \cdot P_{2}}=\frac{\Gamma_{2} \Gamma_{3}}{d_{2} d_{3}} / \frac{\Gamma_{2}}{d_{1} d_{2}} \cdot \frac{\Gamma_{3}}{d_{1} d_{3}}=d_{1}^{2}
$$

and thus $d_{1}=\sqrt{P_{3} / P_{1} \cdot P_{2}}$. Equ. (7) is a foundation of our rest algorithm design.

\section{M-path case}

Suppose there are an arbitrary number of paths between the transmitter and receiver and this number of paths $\mathrm{M}$ is known. In this scenario, we note that Equ. (7) still holds but Equ. (6) does not work because there are many non-zero values and we have no idea which three satisfy Equ. (7). The main tasks of this subsection is then to identify three such DFT amplitudes.

When there are $M$ different paths, the RSS at the receiver can be expressed as (more details are omitted)

$$
s(\lambda)=C \cdot \lambda^{2} \cdot\left(\sum_{m=1}^{M} \frac{\Gamma_{m}}{2 d_{m}^{2}}+\sum_{m \neq m^{\prime}} \frac{\Gamma_{m} \Gamma_{m^{\prime}}}{d_{m} d_{m^{\prime}}} \cos \left(\frac{d_{m}-d_{m^{\prime}}}{\lambda}\right)\right)
$$

where $d_{m}$ and $\Gamma_{m}$ are the m-th path length and reflection coefficient. Applying the DFT method on $s(\lambda)$ we get

$$
\left\{\begin{array}{l}
P(0)=\sum_{m=1}^{M} \frac{\Gamma_{m}}{2 d_{m}^{2}} \\
P\left(d_{m}-d_{m^{\prime}}\right)=\frac{\Gamma_{m} \Gamma_{m^{\prime}}}{d_{m} d_{m^{\prime}}}
\end{array}\right.
$$

Fig. 2 gives an example of $\hat{s}_{n}$ and the DFT amplitude $P(k)$ under a 5-path scenario. According to Equ. (9), at each 
combination of $k=d_{m}-d_{m^{\prime}}$, there will be a non-zero value $P\left(d_{m}-d_{m^{\prime}}\right)=\frac{\Gamma_{m} \Gamma_{m^{\prime}}}{d_{m} d_{m^{\prime}}}$. In total, there are $\left(\begin{array}{l}M \\ 2\end{array}\right)=M(M-1) / 2$ non-zero $P(k)$. We call $P\left(d_{m}-d_{m^{\prime}}\right)$ the DFT amplitude induced by the $d_{m}$ and $d_{m^{\prime}}$ paths. From this number of nonzero $\mathrm{P}(\mathrm{k})$ we need to identify three $\mathrm{P}(\mathrm{k})$ satisfying that, the former two are respectively induced by the $\operatorname{LoS}$ path and a NLoS path, and the last one is induced by the latter two NLoS paths. In other words, the three should have the form as $P\left(d_{1}-d_{m}\right), P\left(d_{1}-d_{m^{\prime}}\right)$, and $P\left(d_{m}-d_{m^{\prime}}\right)$. For this we have,

Lemma 3.1: $\forall m, m^{\prime}>1, P\left(d_{1}-d_{m}\right)>P\left(d_{m^{\prime}}-d_{m}\right)$

We omit all the proof in this paper because of the space limitation.

Theorem 3.2: When $M \geq 3$, the two largest DFT amplitude $P_{1}$ and $P_{2}$ must be induced by the LoS path and a NLoS path respectively.

With this theorem, the identification of three amplitudes for Equ (7) becomes straightforward. The two largest one DFT amplitude $P_{1}$ and $P_{2}$ will satisfy the first two, and the third one $P\left(d_{m}-d_{m^{\prime}}\right)$ can be obtained by

$$
d_{m}-d_{m^{\prime}}=\left(d_{1}-d_{m^{\prime}}\right)-\left(d_{1}-d_{m}\right)=\underset{k}{\arg }\left(P_{1}\right)-\underset{k}{\arg }\left(P_{2}\right)
$$

where $\underset{k}{\arg }\left(P_{i}\right)$ is the index of $P_{i}$. Thus, we have

$$
d_{1}=\sqrt{\frac{P\left(\underset{k}{\arg }\left(P_{1}\right)-\underset{k}{\arg }\left(P_{2}\right)\right) /\left(P_{1} P_{2}\right)}{2}}
$$

Notice that Equ. (10) is an exact express. It will produce optimal solutions when all the input $P_{1}, P_{2}$, and $P\left(\arg \left(P_{1}\right)-\right.$ $\arg \left(P_{2}\right)$ are accurate.

Notice that Equ. (10) is very sensitive to $P(k)$ values, while $P\left(\underset{k}{\arg }\left(P_{1}\right)-\underset{k}{\arg }\left(P_{2}\right)\right)$ the M-path algorithm is typically very small and easy to be impaired by noises. To be robust to the input noise, a key observation is that the large amplitudes (e.g., $P_{1}, P_{2}$ in Equ. (10) are robust while small values (e.g., $\left.P\left(\underset{k}{\arg }\left(P_{1}\right)-\underset{k}{\arg }\left(P_{2}\right)\right)\right)$ are more prone to be impacted. Accordingly, in our robust DFT-based algorithm, we prefer to use large $P(k)$, e.g., the largest $P_{i}$.

$$
\begin{aligned}
d_{1} & =1 / \sqrt{P(0)-\sum_{m=2}^{M} \frac{\Gamma_{m}^{2}}{d_{m}^{2}}} \\
& \approx 1 / \sqrt{P(0)-\sum_{m=2}^{M} P\left(d_{m}-d_{1}\right)^{2} / 2 P(0)}
\end{aligned}
$$

So we utilize the Equ. (11) to solve $d_{1}$ more often. To the limitation of the length of the paper, we omit the derivation. In the Equ. (11), $\mathrm{M}$ is the path number. By Lemma 3.1, we can safely assume that the top M DFT amplitudes $P_{1}, \ldots, P_{M}$ are all induced by the LoS and an NLoS path but not two NLoS paths.

\section{PERformance Evaluations}

In this section, we evaluate the performance of Fredi ranging algorithm through real implementation experiments. We first introduce the experiment setup, and present the experimental results in detail.

\section{A. Experiment setup}

We implement general Fredi algorithm using the GNU USRP2 platform [4]. We select the USRP2 platform as it provides the full control for physical layer information, which is extremely helpful to analyze the experimental data. The RFX5500 daughter boards are employed that support the frequency hopping from $5.0 \mathrm{G}$ to $5.9 \mathrm{G}$. When ranging, the transmitter sends out packets that carry the frequency hopping information. The receiver receives the packets, decodes to synchronization information, and measure the RSS of the packets in the meanwhile.

In our experiments, up to 500 measurements (i.e., $n=500$ ) are measured for one ranging, and in later sections we will investigate the impact of $N$. Each RSS value is averaged from five runs, and all the data are transmitted back to a PC and processed in a centralized manner.

To investigate the effectiveness of Fredi, we also implement the traditional RSS-based ranging in the same testbed and make comparisons with Fredi. In our designs, RSS measurements are the only input and no other information is employed. To make a fair comparison, we do not use any other information in traditional methods either. Thus, traditional RSS-based algorithms can do very little. Given the hardwaredependent parameter $C$ as in Fredi design, we adopt the open space model (Equ. (2)) to compute the theoretical RSS, compare the measured RSS values with the theoretical ones, and map the RSS values to the corresponding distances.

In our experiments, we care only one metric, the ranging accuracy. Given the real distance $\hat{d}_{1}$, we are interested in the measured distance $d_{1}$, and the error in between.

\section{B. Effectiveness of DFT-based algorithm and impact of $N$}

In the set of experiments, we investigate the the FREDI algorithm performance on 500 measurements of frequencies and the impact of the number of measurement $N$ on the ranging accuracy. Notice that when $N=1$, it becomes the traditional method.

Fig. 3 shows the results by the FREDI algorithm under an indoor environment. The usrp2 will play all the ability on the ranging accuracy when $N=500$, Within $4 \mathrm{~m}$, the accuracy is within $0.1 \mathrm{~m}$, and within $6 \mathrm{~m}$, the range error is no more than $0.5 \mathrm{~m}$. Beyond 7 meters, there will be a sharp degradation of the accuracy to 1 meters. From the results of the impact of the number of measurement $N$, we find that when $N$ is less than 16, the ranging error is quite high, and more importantly, the measured distance is not a monotonic function of the real distance. Such performance can hardly be accepted in practice. For scenarios of $N \geqslant 16$, the performance greatly improves. We further notice that there is not a great incentive to further increase $N$. The performance gap between $N=16$ and $N=$ 500 is less than 10 percent.

\section{Comparisons with traditional methods}

In this part, we make comparisons between Fredi and the traditional RSS-based ranging. We implement the traditional RSS ranging as Sec. IV-A described. Fig. 4 shows the ranging 
accuracy of traditional RSS in the same indoor environment. We use an errorbar to depict the result.

We observe that when the transmitter and receiver are very close to each other, i.e., $d_{1}=2$, traditional methods can perform pretty well. The ranging accuracy is within $0.1 \mathrm{~m}$ and the results are very stable. This is because in this close distance, the LoS path waves will exhibit preeminent advantages by its shorter length and no reflection lose. In addition, at this distance, there are not too many, if any, NLoS paths, and thus the impact of multipath effect is little. Beyond three meters, the advantages of LoS path waves becomes weakened, and more NLoS paths appear. As the distance further increases, the performance of traditional methods further degrade. We even observe a measured distance as $80 \mathrm{~m}$ when the real distance is only $5 \mathrm{~m}$.

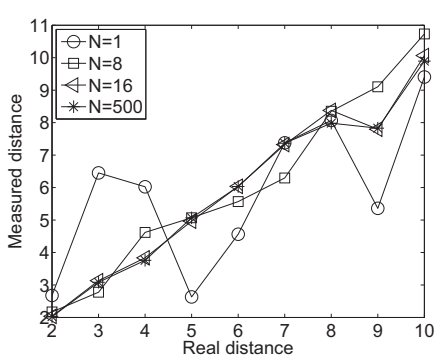

Fig. 3. Impact of $\mathrm{N}$

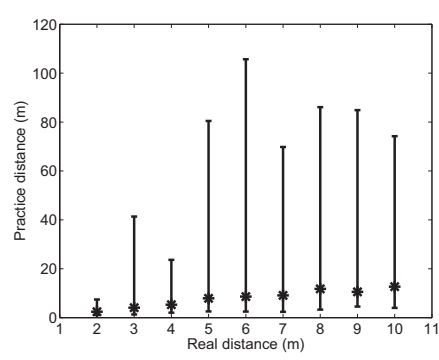

Fig. 4. Traditional RSS ranging

\section{RELATED WORK}

RSS-based ranging has been an active research area for decades of years. Because of the multi-path and many other practical issues, the RSS cannot be simply converted to the ranging and various techniques have been proposed. We classify the existing methods into two categories, namely modelbased and fingerprint-based.

In the model-based approaches, a radio propagation model is established to predict the RSS at the different distances. As the simple radio propagation model performs badly in practice, various new models have been proposed. For example Lim et al. [3] used the LDPL model, and ARIADNE [2] adopted a ray-tracking model based on floor maps. In these works, the more inputs for building models were obtained a larger deployment of measurement nodes (e.g., AP). As the model parameters are quite different for differen nodes, these approaches are limited in dynamic environments. None of them exploited the frequency diversity yet.

In the fingerprint-based approaches (e.g., RADAR [1] and PinLoc [9]), an RF signal map is build in a pre-deployment stage. Users match the measured RSS with this map to obtain their locations. Fingerprint-based approaches can effectively address the multipath issue but are limited to static environments where the environment parameters do not change. In dynamic environments, the map has to be rebuild constantly, which is often too costly. Compared with fingerprint based approaches, Fredi does not require excessive pre-deployment efforts.
Besides the RSS-based ranging, there are some other indoor ranging techniques such as the Angle-of-Arrival (AoA) [8], Time-of-Arrival such as Beepbeep [5], Time-Differenceof-Arrival such as the Cricket [6], light-based [10]. These approaches are always based on the assumption of single LoS path and they often requires special hardware to obtain the required information. To the contrast, Fredi does not require any special hardware, physical layer information, or interactions from the users.

\section{CONClusion ANd Future Work}

Frequency diversity provides a powerful tool to address the multipath effect. By frequency diversity, RSS measurements at different frequencies will be fundamentally different, which help us to build a mathematical model to figure out all the dynamic factors. Compared with the traditional methods to deal with multipath effect and radio interference, Fredi is simple and easy to employ. The only input is the RSS measurement, no physical layer information is needed, and no cooperation between nodes are needed. The only requirement is to quick measure the RSS at different frequencies so that the dynamic factors will not likely to change during the measurement. The Fredi has a stable performance in the environment of radio interference from other sources, as the DFT can dilute these interferences in the frequency domain. By current off-theshelf hardware, this is a reasonable requirement. Experimental results show that attractive performance of Fredi.

\section{ACKNOWLEDGEMENTS}

This research was supported in part by China NSFC Grant 61170247, National Science and Technology Major Project 2013ZX010033002-003, and National Key Technology Research and Development Program 2012BAH07B00.

\section{REFERENCES}

[1] P. Bahl and V. Padmanabhan. Radar: An in-building rf-based user location and tracking system. In Proc. of IEEE INFOCOM. Ieee, 2000.

[2] Y. Ji, S. Biaz, S. Pandey, and P. Agrawal. Ariadne: a dynamic indoor signal map construction and localization system. In Proc. of $A C M$ Mobisys, pages 151-164. ACM, 2006.

[3] H. Lim, L. Kung, J. Hou, and H. Luo. Zero-configuration, robust indoor localization: Theory and experimentation. In Proc. of IEEE Infocom, pages 1-12. Ieee, 2006.

[4] E. R. LLC. Universal soft radio peripheral, 2006.

[5] C. Peng, G. Shen, Y. Zhang, Y. Li, and K. Tan. Beepbeep: a high accuracy acoustic ranging system using cots mobile devices. In Proc. of ACM Sensys, pages 1-14. ACM, 2007.

[6] N. Priyantha, A. Chakraborty, and H. Balakrishnan. The cricket locationsupport system. In Proc. of ACM Mobicom, pages 32-43. ACM, 2000.

[7] H. Rahul, F. Edalat, D. Katabi, and C. Sodini. Fara: Frequency-aware rate adaptation and mac protocols. In Proc. of ACM MOBICOM, pages 193-204. ACM, 2009.

[8] P. Rong and M. Sichitiu. Angle of arrival localization for wireless sensor networks. In Proc. of IEEE SECON, volume 1, pages 374-382. Ieee, 2006.

[9] S. Sen, R. Choudhury, B. Radunovic, and T. Minka. Precise indoor localization using phy layer information. In Proceedings of the 10th ACM Workshop on Hot Topics in Networks, page 18. ACM, 2011.

[10] R. Stoleru, T. He, J. A. Stankovic, and D. Luebke. A high-accuracy, lowcost localization system for wireless sensor networks. In Proceedings of ACM SenSys, 2005.

[11] Z. Yang and Y. Liu. Quality of trilateration: Confidence-based iterative localization. IEEE TPDS, 21(5):631-640, 2010. 